\title{
Relations Among Bell Polynomials, Central Factorial Numbers, and Central Bell Polynomials
}

\author{
Feng Qi and Bai-Ni Guo*
}

\begin{abstract}
In the note, by virtue of the Faà di Bruno formula and two identities for the Bell polynomials of the second kind, the authors derive three relations among the Bell polynomials, central factorial numbers of the second kind, and central Bell polynomials.
\end{abstract}

Keywords: Bell polynomial; central factorial number of the second kind; central Bell polynomial; Bell polynomial of the second kind; Fà̀ di Bruno formula.

AMS Subject Classification (2010): Primary 11B83; Secondary 11B75, $33 B 10$.

${ }^{*}$ Corresponding author

\section{Preliminaries}

The Bell numbers $B_{k}$ for $k \geq 0$ can be generated $[4,7,12]$ by

$$
e^{e^{t}-1}=\sum_{k=0}^{\infty} B_{k} \frac{t^{k}}{k !}=1+t+t^{2}+\frac{5}{6} t^{3}+\frac{5}{8} t^{4}+\frac{13}{30} t^{5}+\frac{203}{720} t^{6}+\frac{877}{5040} t^{7}+\cdots
$$

As a generalization of the Bell numbers $B_{k}$ for $k \geq 0$, the Bell polynomials $T_{k}(x)$ for $k \geq 0$ can be generated [8$10,15,17]$ by

$$
\begin{gathered}
e^{x\left(e^{t}-1\right)}=\sum_{k=0}^{\infty} T_{k}(x) \frac{t^{k}}{k !}=1+x t+\frac{1}{2} x(x+1) t^{2}+\frac{1}{6} x\left(x^{2}+3 x+1\right) t^{3} \\
+\frac{1}{24} x\left(x^{3}+6 x^{2}+7 x+1\right) t^{4}+\frac{1}{120} x\left(x^{4}+10 x^{3}+25 x^{2}+15 x+1\right) t^{5}+\cdots
\end{gathered}
$$

The polynomials $T_{k}(x)$ for $k \geq 0$ are also called [11,18] the Touchard polynomials or the exponential polynomials. It is clear that $T_{k}(1)=B_{k}$.

The central factorial numbers of the second kind $T(n, k)$ for $n \geq k \geq 0$ can be generated [1,6] by

$$
\frac{1}{k !}\left(2 \sinh \frac{t}{2}\right)^{k}=\sum_{n=k}^{\infty} T(n, k) \frac{t^{n}}{n !},
$$

where

$$
\sinh t=\frac{e^{t}-e^{-t}}{2}
$$

is the hyperbolic sine function.

The central Bell polynomials $B_{k}^{(c)}(x)$ for $k \geq 0$ can be generated [5] by

$$
\exp \left(2 x \sinh \frac{t}{2}\right)=\sum_{k=0}^{\infty} B_{k}^{(c)}(x) \frac{t^{k}}{k !} .
$$

Received : 16-05-2019, Accepted : 04-10-2019 
In this note, by virtue of the Faà di Bruno formula and two identities for the Bell polynomials of the second kind, we will discuss relations among the Bell polynomials $T_{k}(x)$, central factorial numbers of the second kind $T(n, k)$, and central Bell polynomials $B_{k}^{(c)}(x)$.

\section{Lemmas}

The Bell polynomials of the second kind, denoted by $\mathrm{B}_{n, k}\left(x_{1}, x_{2}, \ldots, x_{n-k+1}\right)$ for $n \geq k \geq 0$, are defined $[2,3$, $11,13,15-17$ by

$$
\mathrm{B}_{n, k}\left(x_{1}, x_{2}, \ldots, x_{n-k+1}\right)=\sum_{\substack{1 \leq i \leq n-k+1 \\ \ell_{i} \in\{0\} \cup \mathbb{N} \\ \sum_{i=1}^{n-k+1} i \ell_{i}=n \\ \sum_{i=1}^{n-k+1} \ell_{i}=k}} \frac{n !}{\prod_{i=1}^{n-k+1} \ell_{i} !} \prod_{i=1}^{n-k+1}\left(\frac{x_{i}}{i !}\right)^{\ell_{i}} .
$$

For proving our main results, we need the following lemmas.

Lemma 2.1 ([2, 3, 14, 19-21]). The Faà di Bruno formula can be described in terms of $\mathrm{B}_{n, k}\left(x_{1}, x_{2}, \ldots, x_{n-k+1}\right)$ by

$$
\frac{\mathrm{d}^{n}}{\mathrm{~d} x^{n}} f \circ h(x)=\sum_{k=0}^{n} f^{(k)}(h(x)) \mathrm{B}_{n, k}\left(h^{\prime}(x), h^{\prime \prime}(x), \ldots, h^{(n-k+1)}(x)\right) .
$$

For $n \geq k \geq 0$, the Bell polynomials of the second kind $\mathrm{B}_{n, k}\left(x_{1}, x_{2}, \ldots, x_{n-k+1}\right)$ satisfy the identity

$$
\mathrm{B}_{n, k}\left(a b x_{1}, a b^{2} x_{2}, \ldots, a b^{n-k+1} x_{n-k+1}\right)=a^{k} b^{n} \mathrm{~B}_{n, k}\left(x_{1}, x_{2}, \ldots, x_{n-k+1}\right)
$$

is valid, where $a, b \in \mathbb{C}$.

Lemma $2.2([14,19,21])$. For $n \geq k \geq 0$, the Bell polynomials of the second kind $\mathrm{B}_{n, k}\left(x_{1}, x_{2}, \ldots, x_{n-k+1}\right)$ satisfy the closed formula

$$
\mathrm{B}_{n, k}\left(1,0,1, \ldots, \frac{1-(-1)^{n-k+1}}{2}\right)=\frac{1}{2^{k} k !} \sum_{\ell=0}^{k}(-1)^{\ell}\left(\begin{array}{c}
k \\
\ell
\end{array}\right)(k-2 \ell)^{n} .
$$

\section{Main results and their proofs}

Now we are in a position to state and prove our main results.

Theorem 3.1. For $k \geq 0$, we have

$$
\begin{aligned}
& B_{k}^{(c)}(x)=\frac{(-1)^{k}}{2^{k}} \sum_{\ell=0}^{k}(-1)^{\ell}\left(\begin{array}{l}
k \\
\ell
\end{array}\right) T_{\ell}(x) T_{k-\ell}(-x), \\
& B_{k}^{(c)}(x)=\sum_{\ell=0}^{k} T(k, \ell) x^{\ell},
\end{aligned}
$$

and

$$
\sum_{\ell=0}^{k} T(k, \ell) x^{\ell}=\left(-\frac{1}{2}\right)^{k} \sum_{\ell=0}^{k}(-1)^{\ell}\left(\begin{array}{l}
k \\
\ell
\end{array}\right) T_{\ell}(x) T_{k-\ell}(-x) .
$$

Proof. Utilizing (1.2) and (1.1) in sequence, it follows that

$$
\begin{gathered}
\exp \left(2 x \sinh \frac{t}{2}\right)=e^{x\left(e^{t / 2}-1\right)} e^{-x\left(e^{-t / 2}-1\right)}=\left[\sum_{k=0}^{\infty} \frac{T_{k}(x)}{2^{k}} \frac{t^{k}}{k !}\right]\left[\sum_{k=0}^{\infty} \frac{T_{k}(-x)}{(-2)^{k}} \frac{t^{k}}{k !}\right] \\
=\sum_{k=0}^{\infty}\left[\sum_{\ell=0}^{k}(-1)^{\ell}\left(\begin{array}{l}
k \\
\ell
\end{array}\right) T_{\ell}(x) T_{k-\ell}(-x)\right]\left(-\frac{1}{2}\right)^{k} \frac{t^{k}}{k !} .
\end{gathered}
$$

Comparing this with (1.3) yields (3.1). 
From (1.3), (2.1), (2.2), and (2.3) in sequence, it follows that

$$
\begin{aligned}
B_{k}^{(c)}(x) & =\lim _{t \rightarrow 0} \frac{\mathrm{d}^{k}}{\mathrm{~d} t^{k}}\left[\exp \left(2 x \sinh \frac{t}{2}\right)\right] \\
& =\lim _{t \rightarrow 0} \sum_{\ell=0}^{k}\left(e^{2 x u}\right)^{(\ell)} \mathrm{B}_{k, \ell}\left(\left(\sinh \frac{t}{2}\right)^{\prime},\left(\sinh \frac{t}{2}\right)^{\prime \prime}, \ldots,\left(\sinh \frac{t}{2}\right)^{(n-k+1)}\right) \\
& =\lim _{t \rightarrow 0} \sum_{\ell=0}^{k}(2 x)^{\ell} e^{2 x u} \mathrm{~B}_{k, \ell}\left(\frac{1}{2} \cosh \frac{t}{2}, \frac{1}{2^{2}} \sinh \frac{t}{2}, \ldots, \frac{1}{2^{n-k+1}} \sinh ^{(n-k+1)} \frac{t}{2}\right) \\
& =\sum_{\ell=0}^{k} \frac{(2 x)^{\ell}}{2^{k}} \mathrm{~B}_{k, \ell}\left(1,0,1,0, \ldots, \frac{1-(-1)^{n-k+1}}{2}\right) \\
& =\sum_{\ell=0}^{k} \frac{(2 x)^{\ell}}{2^{k}} \frac{1}{2^{\ell} \ell !} \sum_{m=0}^{\ell}(-1)^{m}\left(\begin{array}{c}
\ell \\
m
\end{array}\right)(\ell-2 m)^{k} \\
& =\sum_{\ell=0}^{k} \frac{x^{\ell}}{\ell !} \sum_{m=0}^{\ell}(-1)^{m}\left(\begin{array}{c}
\ell \\
m
\end{array}\right)\left(\frac{\ell}{2}-m\right)^{k} \\
& =\sum_{\ell=0}^{k} T(k, \ell) x^{\ell},
\end{aligned}
$$

where $u=u(t)=\sinh \frac{t}{2}$. Consequently, we derive the relation (3.2).

Combining the relation (3.1) with the equation (3.2) leads to the equality (3.3). The proof of Theorem 3.1 is complete.

\section{References}

[1] Butzer, P. L., Schmidt, M., Stark, E. L. and Vogt, L., Central factorial numbers; their main properties and some applications, Numer. Funct. Anal. Optim. 10 (1989), no. 5-6, 419-488; available online at https: / / doi .org / $10.1080 / 01630568908816313$.

[2] Charalambides, C. A., Enumerative Combinatorics, CRC Press Series on Discrete Mathematics and its Applications. Chapman \& Hall/CRC, Boca Raton, FL, 2002.

[3] Comtet, L., Advanced Combinatorics: The Art of Finite and Infinite Expansions, Revised and Enlarged Edition, D. Reidel Publishing Co., 1974; available online at https://doi .org/10.1007/978-94-010-2196-8.

[4] Guo, B.N. and Qi, F., An explicit formula for Bell numbers in terms of Stirling numbers and hypergeometric functions, Glob. J. Math. Anal. 2 (2014), no. 4, 243-248; available online at http://dx.doi.org/10.14419/g jma. v2i 4.3310.

[5] Kim, D. S., Jang, G.W., Dolgy, D. V. and Kim, T., An expression for central Bell polynomials, Adv. Stud. Contemp. Math. 29 (2019), no. 2, 257-262; available online at http: / / dx. doi . org/10.17777/as cm2019.29.2.257.

[6] Merca, M., Connections between central factorial numbers and Bernoulli polynomials, Period. Math. Hungar. 73 (2016), no. 2, 259-264; available online at https://doi.org/10.1007/s10998-016-0140-5.

[7] Qi, F., An explicit formula for the Bell numbers in terms of the Lah and Stirling numbers, Mediterr. J. Math. 13 (2016), no. 5, 2795-2800; available online at https://doi.org/10.1007/s00009-015-0655-7.

[8] Qi, F., Integral representations for multivariate logarithmic polynomials, J. Comput. Appl. Math. 336 (2018), 54-62; available online at https://doi.org/10.1016/j.cam.2017.11.047.

[9] Qi, F., On multivariate logarithmic polynomials and their properties, Indag. Math. (N.S.) 29 (2018), no. 5, 1179-1192; available online at https://doi.org/10.1016/j.indag.2018.04.002. 
[10] Qi, F., Simplifying coefficients in differential equations related to generating functions of reverse Bessel and partially degenerate Bell polynomials, Bol. Soc. Paran. Mat. 39 (2021), no. 4, in press; available online at http: / dx . doi. org/10.5269/bspm. 41758 .

[11] Qi, F., Some inequalities and an application of exponential polynomials, Math. Inequal. Appl. 22 (2019), in press; available online at https://doi.org/10.20944/preprints201708.0079.v2.

[12] Qi, F., Some inequalities for the Bell numbers, Proc. Indian Acad. Sci. Math. Sci. 127 (2017), no. 4, 551-564; available online at https://doi.org/10.1007/s12044-017-0355-2.

[13] Qi, F., Čerňanová, V. and Semenov, Y. S., Some tridiagonal determinants related to central Delannoy numbers, the Chebyshev polynomials, and the Fibonacci polynomials, Politehn. Univ. Bucharest Sci. Bull. Ser. A Appl. Math. Phys. 81 (2019), no. 1, 123-136.

[14] Qi, F. and Guo, B.N., Explicit formulas for special values of the Bell polynomials of the second kind and for the Euler numbers and polynomials, Mediterr. J. Math. 14 (2017), no. 3, Article 140, 14 pages; available online at https://doi.org/10.1007/s00009-017-0939-1.

[15] Qi, F., Lim, D. and Guo, B.N., Explicit formulas and identities for the Bell polynomials and a sequence of polynomials applied to differential equations, Rev. R. Acad. Cienc. Exactas Fís. Nat. Ser. A Mat. RACSAM 113 (2019), no. 1, 1-9; available online at https://doi.org/10.1007/s13398-017-0427-2.

[16] Qi, F., Lim, D. and Yao, Y.H., Notes on two kinds of special values for the Bell polynomials of the second kind, Miskolc Math. Notes 20 (2019), no. 1, 465-474; available online at https: / / doi.org/10.18514/MMN.2019.2635.

[17] Qi, F., Niu, D.W. and Guo, B.N., Some identities for a sequence of unnamed polynomials connected with the Bell polynomials, Rev. R. Acad. Cienc. Exactas Fís. Nat. Ser. A Math. RACSAM 113 (2019), no. 2, 557-567; available online at https://doi.org/10.1007/s13398-018-0494-z.

[18] Qi, F., Niu, D.W., Lim, D. and Guo, B.N., Some properties and an application of multivariate exponential polynomials, HAL archives (2018), available online at https: //hal . archives-ouvertes.fr/hal-01745173.

[19] Qi, F., Niu, D.W., Lim, D. and Yao, Y.H., Special values of the Bell polynomials of the second kind for some sequences and functions, HAL archives (2018), available online at https://hal.archives-ouvertes.fr/ hal-01766566.

[20] Qi, F. and Zheng, M.M., Explicit expressions for a family of the Bell polynomials and applications, Appl. Math. Comput. 258 (2015), 597-607; available online at https://doi.org/10.1016/j .amc.2015.02.027.

[21] Wei, C.F. and Qi, F., Several closed expressions for the Euler numbers, J. Inequal. Appl. 2015, 2015:219, 8 pages; available online at https://doi.org/10.1186/s13660-015-0738-9.

\section{Affiliations}

FENG QI

ADDRESS: Inner Mongolia University for Nationalities, College of Mathematics, Tongliao 028043, Inner Mongolia, China; Tianjin Polytechnic University, School of Mathematical Sciences, Tianjin 300387, China.

E-MAIL: qifeng618@gmail.com

ORCID ID: https://orcid.org/0000-0001-6239-2968

BAI-Ni GUO (CORRESPONDING AUTHOR)

AdDress: Henan Polytechnic University, School of Mathematics and Informatics, Jiaozuo 454010, Henan, China. E-MAIL: bai.ni.guo@gmail.com

ORCID ID: https://orcid.org/0000-0001-6156-2590 